\title{
OPTICALLY DETECTED MAGNETIC RESONANCE OF THE TRYPTOPHAN PHOSPHORESCENT STATE IN NATIVE PROTEINS*
}

\author{
J. Zuclich, D. Schweitzer $\dagger$ and A. H. MAKI \\ Department of Chemistry, University of California, Riverside, California 92502, U.S.A.
}

(Received 18 December 1972; accepted 13 March 1973)

\begin{abstract}
The phosphorescent triplet state of tryptophan has been studied by the method of optically detected magnetic resonance (ODMR) at pumped helium temperatures in zero magnetic field. Only one of the triplet sublevels is found to be significantly radiative; the other two decay radiationlessly. Although the phosphorescence and ODMR decay lifetimes are influenced by spin-lattice relaxation processes at $T=1.3^{\circ} \mathrm{K}$, the lifetime of the radiative level can be estimated as approximately $2 \mathrm{~s}$, whereas the lifetimes of the non-radiative levels are in excess of $10 \mathrm{~s}$. Comparison of the ODMR signals and the phosphorescence spectra has been made for tryptophans in native proteins with the following results: the ODMR signals of the two types of tryptophan sites in horse liver alcohol dehydrogenase can be resolved due to a shift in the $D$ and $E$ values of the respective triplet states; binding of the substrate tri- $\mathrm{N}$-acetylglucosamine to hen lysozyme leads to a considerable narrowing of the phosphorescence peaks and ODMR signals as well as to a shift in the $E$ value of the triplet state.

The following tentative conclusions can be reached: the tryptophan triplet $D$ and $E$ values are measurably affected by the environment of the chromophore in the protein, as are the linewidths of the magnetic resonance transitions. The $|E|$ value is reduced and the magnetic resonance linewidth is increased with increasing exposure of the tryptophan to hydroxylic solvent. Although a considerable part of the width of the magnetic resonance transition can be ascribed to a heterogeneity of environments in the sample, there appears to exist an intrinsic line-broadening process which at present is not understood.
\end{abstract}

\section{INTRODUCTION}

In the past two decades optical spectroscopy has been used in a large number of studies of proteins and their aromatic amino acid constituents. Extensive reviews on the luminescence of aromatic amino acids (Weinryb and Steiner, 1971) and the luminescence of polypeptides and proteins (Longworth, 1971) have recently been published.

With the development in the past few years of the technique of optical detection of magnetic resonance (ODMR) by Sharnoff (1967), Kwiram (1967) and Schmidt et al. (1967), a new optical method has become available for detailed studies of the populating and depopulating kinetics of aromatic triplet states (Schmidt et al., 1971; Burland and Schmidt, 1971; Harris and Hoover, 1971; Harris, 1971; Winscom and Maki, 1971). This method is also referred to in the literature as "phosphorescence microwave double resonance" or PMDR (Tinti et al., 1969).

\footnotetext{
${ }^{*}$ Preliminary results were presented at the Seventeenth Congress Ampere, Turku, Finland, 21-26 August, 1972 (Abstract U2, p. 87).

†Present address: Department of Molecular Physics, Max Planck Institute, Heidelberg, Germany.
}

The populating rates $\left(K_{f}\right)$ and decay rate contants $\left(k_{i}\right)$ for the triplet state energy sublevels, $T_{i}$, are shown in Fig. 1. The spin-lattice relaxation rate constants, $W_{t}$, are strongly temperaturedependent, and in some cases may become negligible compared with the triplet sublevel decay constants at pumped helium temperatures $\left(<2^{\circ} \mathrm{K}\right)$. In these cases, the phosphorescence is composed of three independent emissions from the sublevels $T_{t}$ to the ground state $S_{0}$. Also, the steady-state populations of the sublevels $\left(n_{i}{ }^{0}\right)$ may deviate substantially from the Boltzmann distribution. In fact, in the absence of spin-lattice relaxation, $n_{i}{ }^{0}=K_{i} / k_{i}$, where the $K_{i}$ 's are expected to differ in general because of selective intersystem-crossing pathways, while the $k_{i}$ 's will differ because of differing spin-orbit mixing of singlets with the triplet sublevels. Although triplet state splitting in the absence of an externally applied magnetic field $\left(\sim 0.1 \mathrm{~cm}^{-1}\right)$ is much too small for optical resolution of the three $T_{i} \rightarrow S_{0}$ transitions, the rate parameters for each sublevel nevertheless may be determined by the techniques described by Schmidt et al. (1971), Burland and Schmidt (1971), Harris and Hoover (1971), Harris (1971) and Winscom and Maki (1971). We will present here only those 


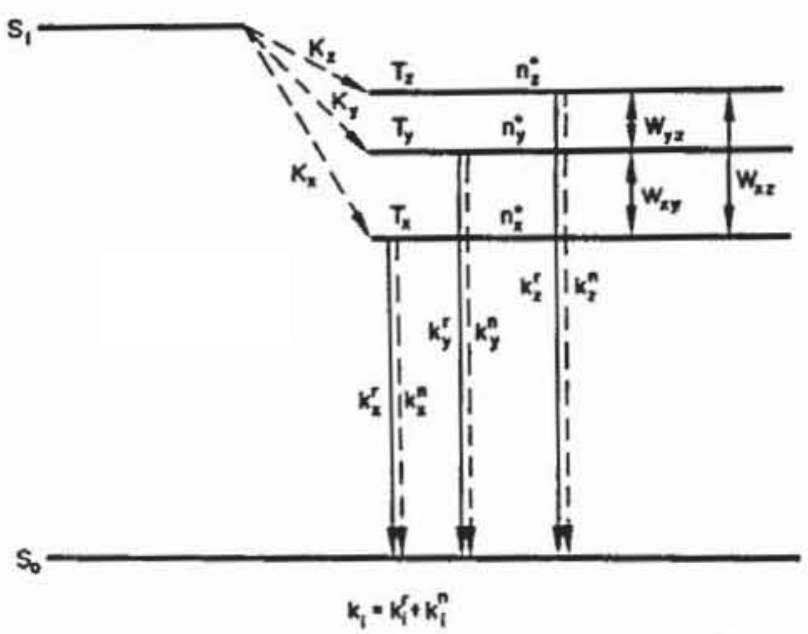

Figure 1. Kinetic rate parameters for the triplet state sublevels $T_{i} . K_{i}$ is the intersystem crossing rate; $k_{i}^{r}$, the radiative decay constant; $k_{i}^{n}$, the non-radiative decay constant, $k_{i}$ the total decay constant; $n_{i}{ }^{\circ}$ the steady-state population; $W_{i}$, the spin-lattice relaxation rate constant between a pair of triplet sublevels. $S_{0}$ and $S_{1}$ are the ground and first excited singlet states, respectively.

aspects of the fast-passage ODMR method (Winscom and Maki, 1971) needed in determination of the triplet state data for the amino acids and proteins reported in this paper.

\section{Fast-passage ODMR}

The rate equation for population of the $i$ th triplet sublevel is given by

$$
\frac{\mathrm{d} n_{i}}{\mathrm{~d} t}=K_{i}-k_{i} n_{i}-\left(W_{i j}+W_{i k}\right) n_{i}+W_{j i} n_{j}+W_{k i} n_{k} .
$$

If spin-lattice relaxation processes are neglected this reduces to

$$
\frac{\mathrm{d} n_{i}}{\mathrm{~d} t}=K_{\mathrm{i}}-k_{i} n_{i}
$$

Under conditions of constant irradiation, the steady-state condition

$$
K_{i}=k_{i} n_{i}^{0}
$$

is seen to hold, where $n_{i}{ }^{0}$ is the steady-state population of the $i$ th sublevel.

Using (3), equation (2) can be integrated to give

$$
n_{i}(t)=c_{i} \mathrm{e}^{-k_{i} t}+n_{i}^{0} .
$$

If the microwave transition between the $i$ th and $j$ th sublevels is saturated at $t=0$ we have

$$
n_{i}(0)=n_{j}(0)=\frac{1}{2}\left(n_{i}^{0}+n_{j}^{0}\right),
$$

so that $c_{i}$ is seen to be given by

$$
c_{i}=\frac{1}{2}\left(n_{j}^{0}-n_{i}{ }^{0}\right) .
$$

The steady-state phosphorescence intensity is given by

$$
I_{0}=S\left[k_{i}^{r} n_{i}^{0}+k_{j}^{r} n_{j}^{0}+k_{k}{ }^{r} n_{k}^{0}\right],
$$

where $k_{i}{ }^{r}$ is the radiative rate constant of $T_{i}$, and $S$ is a constant. Upon saturation of the $i \rightleftarrows j$ transition the phosphorescence intensity becomes

$$
I(t)=S\left[k_{i}^{r} n_{i}(t)+k_{j}^{r} n_{j}(t)+k_{k}^{r} n_{k}^{0}\right] .
$$

Thus, the deviation of the phosphorescence from its steady-state value is just

$$
\begin{aligned}
\Delta I(t)=I(t)-I_{0}=S\left[k_{i}^{r}\left(n_{i}-n_{i}^{0}\right)+k_{j}^{r}\left(n_{j}-n_{j}^{0}\right)\right] \\
=\frac{S}{2}\left(n_{j}^{0}-n_{i}^{0}\right) k_{i}^{r} \mathrm{e}^{-k i t}+\frac{S}{2}\left(n_{i}^{0}-n_{j}^{0}\right) k_{j}^{r} \mathrm{e}^{-k j t} \\
=A \mathrm{e}^{-k_{i} t}-B \mathrm{e}^{-k j t},
\end{aligned}
$$

where

$$
\frac{A}{B}=\frac{k_{i}^{r}}{k_{j}^{r}} .
$$

As seen from (9c), the phosphorescence response upon saturating a microwave transition will consist of a discontinuous jump

$$
\Delta I(0)=\frac{S}{2}\left(k_{i}^{r}-k_{j}^{r}\right)\left(n_{j}^{0}-n_{i}^{0}\right)=A-B,
$$

followed by a decay back to the steady-state phosphorescence intensity, which is a linear combination of two exponentials with lifetimes equal to the total (radiative plus nonradiative) decay constants of the $i$ th and $j$ th sublevels.

Therefore, as illustrated in Fig. 2, the total rate constants $k_{i}$ and $k_{f}$ are determined directly from the phosphorescence response merely by decomposing it into two exponential decays. The relative radiative rate constants, $k_{i}^{r} / k_{j}^{r}=A / B$ are found by extrapolating the two decays back to $t=0$. Furthermore, from (11) and (7) it is seen that $\Delta I(0) / I_{0}=$ (1) $A-B_{(1)} / I_{0}$ involves only ratios of the relative radiative rate constants and the relative steady-state populations. If this quantity is determined for at least two of the three microwave transitions, the equations can be solved for the relative steady-state 


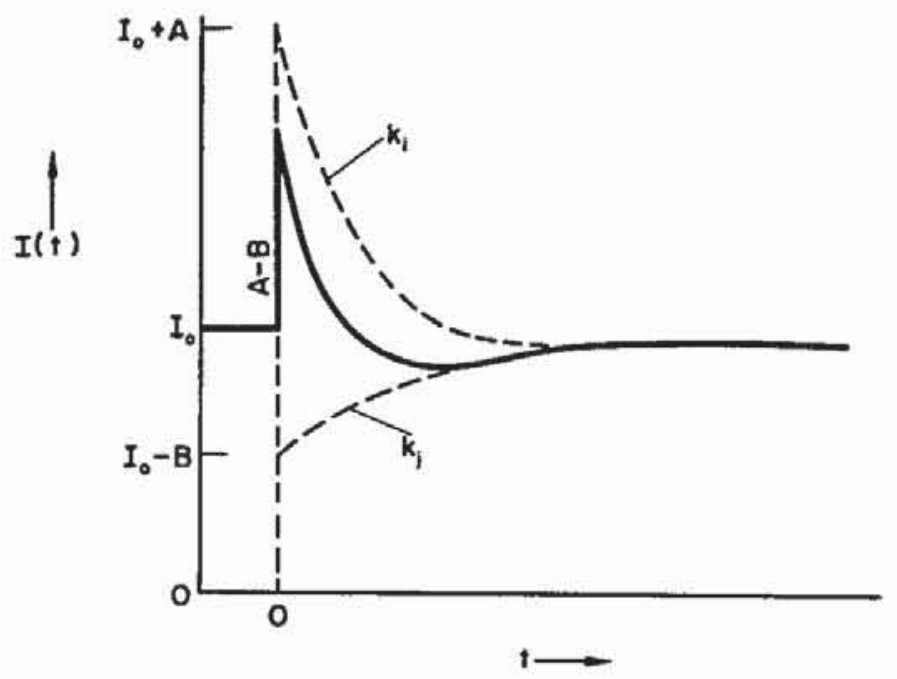

Figure 2. Response of the phosphorescence intensity, $I(t)$, following a saturating microwave pulse (at $t=0$ ) between the $i$ th and $j$ th triplet sublevels.

populations (Winscom and Maki, 1971). The relative populating rates are then found from the steady-state cơnditions, equation (3).

\section{EXPERIMENTAL}

The amino acids and proteins were used as obtained from commercial sources. Trytophan and tyrosine were dissolved at $10^{-3} \mathrm{M}$ in ethylene glycol- $\mathrm{H}_{2} \mathrm{O}(1: 1)$. The protein systems were studied using the same solvent with a suitable amount of protein added to make the final concentration $10^{-3} M$ in tryptophan residues.

The samples were placed in a quartz tube within a slow-wave helix which terminated a coaxial microwave transmission line. This was placed in a liquid helium dewar in which the temperature could be lowered to $\sim 1.25^{\circ} \mathrm{K}$ by pumping. A block diagram of this arrangement is shown in Fig. 3. The samples were excited with a $100 \mathrm{~W}$ PEK mercury lamp filtered with a $1 / 4 \mathrm{~m}$ Bausch \& Lomb monochromator. The phosphorescence was monitored at right angles to the excitation path with a one meter McPherson Model 2051 grating monochromator and detected by a cooled EMI Model 9558QA photomultiplier. A rotating sector was used to eliminate fluorescence and scattered light.

The microwave source was a Hewlett-Packard 8690 B sweep oscillator using plug-in units covering the desired microwave ranges. In some cases a Hughes, Inc., Model $1177 \mathrm{H}$ traveling wave tube amplifier was used and the microwave sweep was synchronized with the on period of a HewlettPackard, Inc., PIN modulator to prevent unnecessary heating of the sample by the amplified power. Repetitive sweeping of the microwaves was synchronized with the sweep of a 1024 channel

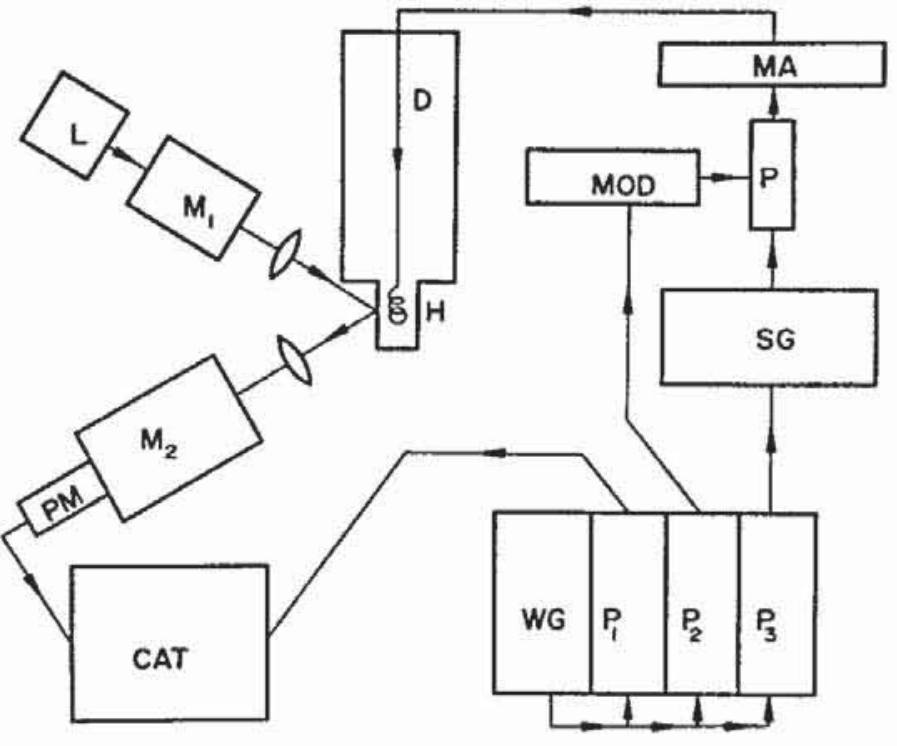

Figure 3. Block diagram of the ODMR apparatus. $L$ is the exciting lamp; $\mathbf{M}_{1}$ and $\mathbf{M}_{2}$, monochromators; $P M$, photomultiplier; $\mathrm{D}$, helium dewar; $\mathrm{H}$, helix containing sample; MA, microwave amplifier. The waveform generator, WG, provides the time base for the pulse units $P_{1}, P_{2}$ and $P_{3}$ which trigger the time averaging computer, CAT, the modulator, MOD, which controls the PIN modulator, $\mathrm{P}$, and the microwave sweep oscillator, SG, respectively.

computer for average transients (CAT) to enhance the signal-to-noise ratio.

\section{RESULTS AND DISCUSSION}

\section{The tryptophan triplet state}

As reported in a preliminary communication (Zuclich et al., 1972) the fast-passage ODMR technique resulted in the observation of optical responses from photoexcited tryptophan when the microwaves were swept through the frequencies $1.736 \mathrm{GHz}$ and $2.449 \mathrm{GHz}$. In each case the response corresponded to an increase in light emission followed by a decay back to the steady-state intensity which appeared to be a single exponential with a lifetime at $1.3^{\circ} \mathrm{K}$ of $1.48 \mathrm{~s} \pm 5$ per cent (Fig. 4). The observation of only a single exponential implies that one of the two sublevels involved in each microwave transition is non-radiative.

The phosphorescence lifetime of tryptophan in frozen glasses at $77^{\circ} \mathrm{K}$ has been reported by many authors (Longworth, 1971) to be approximately $6 \mathrm{~s}$. We find that the phosphorescence decay at $1.3^{\circ} \mathrm{K}$ has two components with lifetimes of 2.5 and $8 \cdot 8 \mathrm{~s}$. In the absence of spin-lattice relaxation (SLR) we would expect to observe the same lifetimes in the phosphorescence decays as are found from the ODMR signals. This is not the case found here. The fact that SLR is non-negligible in this case is further illustrated by varying the temperature and noting the change in both the 


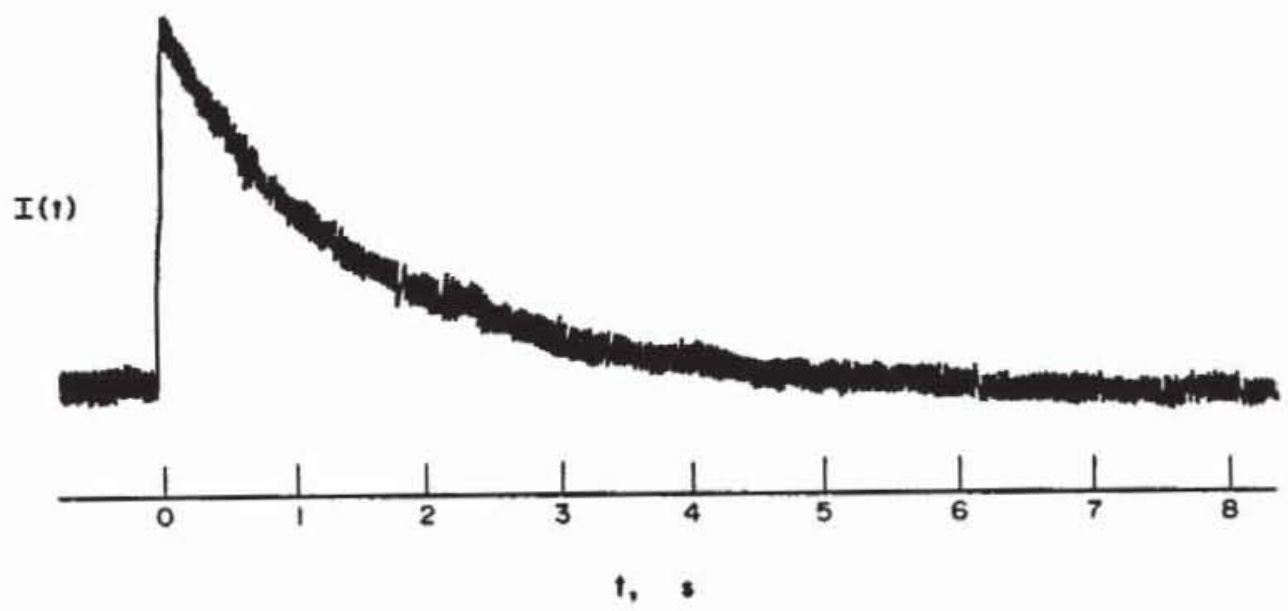

Figure 4. The fast-passage ODMR signal obtained by pumping the $1.736 \mathrm{GHz}$ transition in tryptophan at $1.3^{\circ} \mathrm{K}$. The sample is excited at $290 \mathrm{~nm}$ and the phosphorescence, $I(t)$, monitored at $406 \mathrm{~nm}$. The signal shown is the result of 500 accumulations of the CAT.

ODMR and phosphorescence decay times. At $1.8^{\circ} \mathrm{K}$, for example, the ODMR lifetime is only $1.25 \mathrm{~s}$, while at $4.2^{\circ} \mathrm{K}$ no ODMR signals are observed at all and the phosphorescence decay resembles a single exponential with a decay constant of $\sim 7 \mathrm{~s}$. This indicates that at $4 \cdot 2^{\circ} \mathrm{K}$ the SLR rates have become faster than the lifetime of the triplet sublevels. The presence of SLR always would be expected to shorten the ODMR lifetimes by allowing the triplet sublevels additional pathways to decay back to their steady-state populations. However, SLR tends to average the lifetimes of the phosphorescence decay until at high enough temperatures a single exponential decay is obtained. Thus, we can conclude that the actual lifetime of the short-lived triplet sublevel (we will show later that there is one short-lived sublevel and two long-lived sublevels) of tryptophan is greater than the ODMR lifetime of $1.5 \mathrm{~s}$ but less than the short $2.5 \mathrm{~s}$ component of the phosphorescence decay. Further, the two long-lived sublevels may have lifetimes considerably in excess of $8 \cdot 8 \mathrm{~s}$.

It is seen from equation (1) that in the presence of SLR there is a coupling of the differential equations for the time-dependence of the triplet sublevel populations. This coupled set of differential equations cannot be solved exactly although an approximate treatment has been reported recently by Schweitzer et al. (1973). We can only estimate, therefore, that the short-lived sublevel has a lifetime of $\sim 2 \mathrm{~s}$ and the long-lived sublevels have lifetimes in excess of $10 \mathrm{~s}$.

From the two ODMR signals, the zero-field splitting parameters are calculated to be $|D| / h c=$ $0.0988 \mathrm{~cm}^{-1}$ and $|E| / h c=0.0408 \mathrm{~cm}^{-1}$, in close agreement with the values reported by Zuclich (1970a), using conventional ESR. The third ODMR signal which is expected at $\sim 4.2 \mathrm{GHz}$ is not observed. This feature combined with the fact that the two observed ODMR signals have single exponential decays leads us to conclude that there is at least one and possibly two non-radiative triplet sublevels. If two of the sublevels were radiative, they would have to have the same decay constant within experimental error, as well as either the same steady-state populations or radiative rate constants.

In order to distinguish between these two possibilities we carried out experiments in which the tryptophan was excited by a xenon flashlamp and one of the ODMR transitions was kept continuously saturated. In this case after several lifetimes of a radiative sublevel, that sublevel would have lost most of its population by radiative decay and the non-radiative sublevel connected with it by the continuous microwave saturation would also be drained of population. Thus, if there were two radiative sublevels, all three sublevels would be emptied after three or four radiative lifetimes and we could get no further response by pumping the second ODMR transition. Instead, we find that after a delay of $8 \mathrm{~s}$ or more we get approximately the same optical response from a microwave sweep through one transition with or without continuous saturation of the other transition. We can thus conclude that the tryptophan triplet has one radiative sublevel with $\mathrm{k}^{-1} \sim 2 \mathrm{~s}$ and two nonradiative sublevels with $\mathrm{k}^{-1} \geqslant 10 \mathrm{~s}$. The radiative sublevel is the intermediate one in energy.

The percentage changes in light upon saturation of the microwave transitions are small but by measuring these we found the relative steady-state populations of the sublevels to be $N_{x}: N_{y}: N_{z}=$ $29 \cdot 3: 34 \cdot 7: 36 \cdot 0$. A Boltzmann distribution at $1 \cdot 3^{\circ} \mathrm{K}$ 
would have resulted in $N_{x}: N_{y}: N_{z}=33 \cdot 5: 30 \cdot 7$ : $35 \cdot 8$.

The triplet state data obtained for tryptophan is summarized in Fig. 5(a). The $T_{z}$ triplet sublevel is associated with the principal magnetic axis perpendicular to the aromatic plane, as determined by an ESR magnetophotoselection experiment (Zuclich, 1970a). The molecular orientation of the in-plane principal axes has not been established experimentally but has been calculated (Zuclich, 1970b) to be as shown in Fig. 5(b).

\section{The tyrosine triplet state}

A solution of $10^{-3} M$ tyrosine in ethylene glycol- $\mathrm{H}_{2} \mathrm{O}(1: 1)$ also gave two ODMR signals at $1 \cdot 3^{\circ} \mathrm{K}$. A signal at $2.20 \mathrm{GHz}$ represented a small decrease in light whereas a much stronger signal is observed at $5.59 \mathrm{GHz}$ corresponding to an increase in light which decays with a single exponential whose lifetime is $0.84 \mathrm{~s}$. The zero-field splitting parameters $|D| / h c=0.130 \mathrm{~cm}^{-1}$ and $|E| / h c=$ $0.056 \mathrm{~cm}^{-1}$ again agree closely with those found by conventional ESR spectroscopy (Zuclich, 1970a).

The tyrosine phosphorescence is at most a small component of the total phosphorescence of each protein we have examined and in no case have we seen more than one tyrosine ODMR signal from a

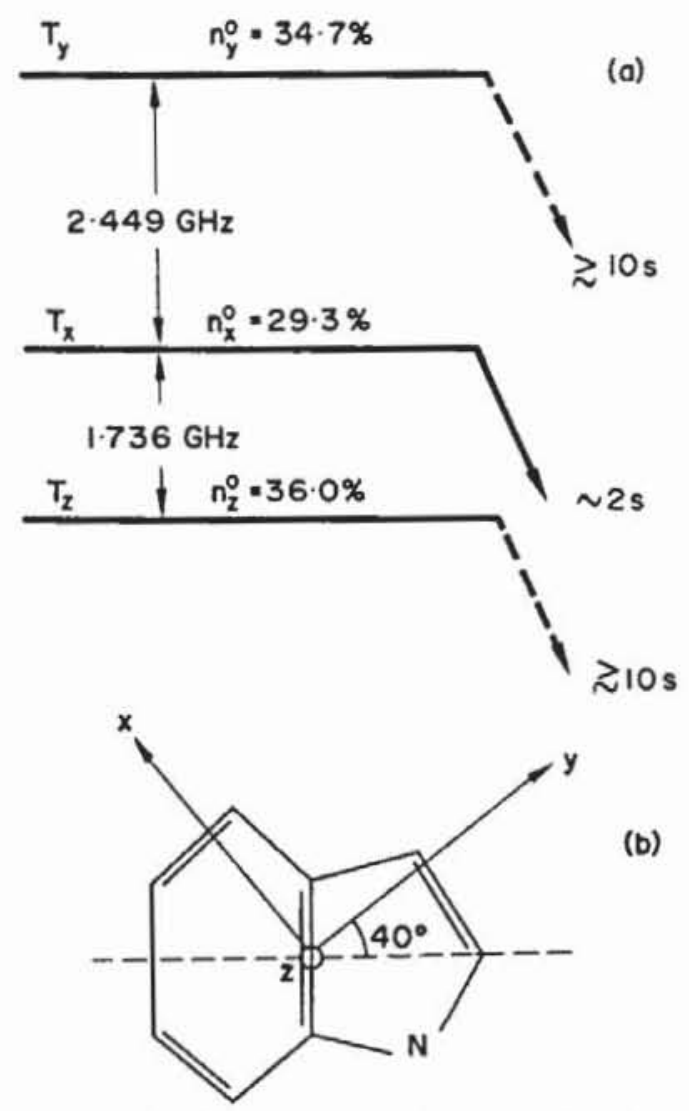

Figure 5. (a) Triplet state data for tryptophan in ethylene glycol- $\mathrm{H}_{2} \mathrm{O}$ as determined by ODMR. (b) The principal magnetic axes of indole. given protein. Therefore, we will not treat tyrosine further in this report.

\section{Tryptophan residues in proteins}

Having determined the triplet state kinetic data of free tryptophan, we then studied the tryptophan phosphorescence of a number of proteins. The main objectives were to resolve different tryptophan sites of each protein by ODMR and to monitor changes at a given site by measuring resulting differences in parameters such as zero-field splitting, ODMR lifetimes, and linewidths.

As a favorable case for resolving the different tryptophan sites of a protein, we chose the enzyme horse liver alcohol dehydrogenase (HLAD, Worthington Biochemical Corp.). This protein has a molecular weight of 80,000 and is composed of two identical subunits, each containing only two tryptophan residues. The phosphorescence spectrum, Fig. 6, has been interpreted by Purkey and Galley (1970) as a superposition of two tryptophan spectra whose origins are shifted by $\sim 6 \mathrm{~nm}$ from each other. Emission studies by Purkey and Galley (1970) have shown that the blue shifted tryptophan emission (tryptophan 1) is due to residues in a polar environment which are freely exposed to the solvent medium while the red shifted emission is from tryptophan (tryptophan 2) in a less polar medium and effectively shielded from the solvent. (The higher polarizability of the interior of the protein molecule provides an explanation for the red shift).

Excitation at $290 \mathrm{~nm}$ results in emission from both tryptophans with about equal intensity. Observing the emission at $405 \mathrm{~nm}$, to the blue of the onset of the red-shifted emission, we can carry out ODMR experiments on tryptophan 1 . With an excitation wavelength of $305 \mathrm{~nm}$, only tryptophan 2 is excited and the ODMR results can be obtained without interference from the blue emission by observing at $410 \mathrm{~nm}$.

The ODMR results are summarized in Table 1. The most striking changes are in the frequencies of the microwave transitions and their linewidths. There did not appear to be any appreciable difference in the triplet sublevel lifetimes or their relative populations. The narrower linewidths of tryptophan 2 are consistent with a more homogenous environment as would be expected at an interior site of the protein. It should be pointed out that in zero field, magnetic resonance linewidths the order of $100 \mathrm{MHz}$ can hardly be caused by any factor except a distribution of $D$ and/or $E$ values among the protein molecules of the sample. 


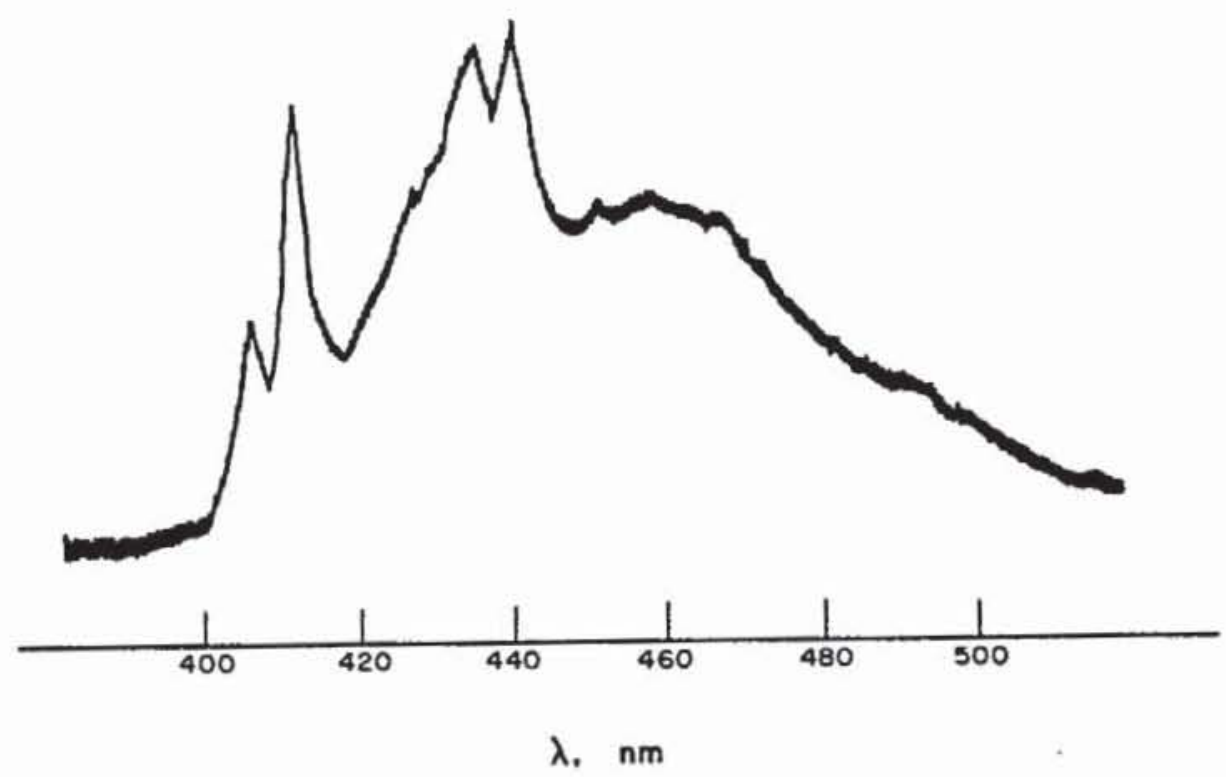

Figure 6. Phosphorescence spectrum of HLAD at $1 \cdot 3^{\circ} \mathrm{K}$ with an exciting wavelength of $295 \mathrm{~nm}$.

Table 1. Data for the tryptophan phosphorescent state in proteins

\begin{tabular}{|c|c|c|c|c|c|}
\hline & \multirow[b]{3}{*}{$\begin{array}{l}\text { Tryptophan } \\
\text { (EG: } \mathrm{H}_{2} \mathrm{O} \text { ) }\end{array}$} & \multicolumn{4}{|c|}{ Protein } \\
\hline & & \multicolumn{2}{|c|}{$\operatorname{HLAD}\left(\mathrm{EG}: \mathrm{H}_{2} \mathrm{O}\right)$} & \multirow[b]{2}{*}{$\begin{array}{l}\text { Lysozyme } \\
\text { (cryst.) }\end{array}$} & \multirow{2}{*}{$\begin{array}{l}\text { Lysozyme- } \\
\text { tri-NAG } \\
\text { (cryst.) }\end{array}$} \\
\hline & & Tryp 1 & Tryp 2 & & \\
\hline Phosphorescence & $406 \cdot 1$ & $406 \cdot 0$ & $411 \cdot 8$ & $415 \cdot 4$ & 415.6 \\
\hline \multicolumn{5}{|l|}{$|D|-|E|$ Transition } & $430 \cdot 4,444 \cdot 2$ \\
\hline Frequency $(\mathrm{GHz})$ & 1.736 & 1.793 & 1.667 & 1.604 & 1.559 \\
\hline Width (MHz) & $165 \cdot 0$ & $125 \cdot 0$ & $75 \cdot 0$ & 145.0 & 85.0 \\
\hline Decay time (s)* & 1.42 & 1.62 & 1.52 & 0.83 & 0.84 \\
\hline \multicolumn{6}{|l|}{$2|E|$ Transition } \\
\hline Frequency $(\mathrm{GHz})$ & $2 \cdot 449$ & $2 \cdot 490$ & $2 \cdot 521$ & $2 \cdot 565$ & 2.667 \\
\hline Width (MHz) & $345 \cdot 0$ & $175 \cdot 0$ & $125 \cdot 0$ & $360 \cdot 0$ & $225 \cdot 0$ \\
\hline Decay time (s)* & 1.55 & 1.60 & 1.66 & 0.87 & 1.02 \\
\hline \multicolumn{6}{|l|}{ Zero-field splitting $\dagger$} \\
\hline$|D| / h c\left(\mathrm{~cm}^{-1}\right)$ & $0.0988(6)$ & $0.1013(4)$ & $0.0977(3)$ & $0.0963(7)$ & $0.0965(4)$ \\
\hline$|E| / h c\left(\mathrm{~cm}^{-1}\right)$ & $0.0408(6)$ & $0.0415(3)$ & $0.0421(2)$ & $0.0428(6)$ & $0.0445(3)$ \\
\hline
\end{tabular}

*Uncertainty in ODMR decay time is $\pm 0.1 \mathrm{~s}$.

†Uncertainty in last figure given in parentheses. $T=1 \cdot 3^{\circ} \mathrm{K}$.

Hyperfine and quadrupole interactions would not be expected to contribute more than a few $\mathrm{MHz}$ to the linewidth. Tryptophan 1, in turn, is in a more homogenous environment than free tryptophan and hence has considerably narrower lines than trytophan completely exposed to the solvent medium.

Another question which one can pose is whether it is possible to monitor changes at a given site when the protein undergoes a biologically significant structural change. In order to test this question we examined crystals of the enzyme lysozyme as well as a crystalline complex of the protein with the substrate tri- $\mathrm{N}$-acetylglucosamine (tri-NAG). The uncharged hen lysozyme was purchased from Schwarz/Mann, Inc. The substrate was kindly donated by Professor John A. Rupley. The lysozyme and lysozyme-substrate crystals were grown from slightly acidic solutions following the method described by Alderton (1946).

Lysozyme has a molecular weight of 14,300 and contains six tryptophan residues per molecule. 
However, Imoto et al. (1972) have shown that the fluorescence emission and therefore presumably the phosphorescence of lysozyme originates almost entirely from two tryptophans (residues 62 and 108) which are situated very close to the active site of the enzyme. The three dimensional structures of lysozyme and the enzyme-substrate complex have been determined by Blake et al. (1967) and from the resulting spacial pictures (Dickerson and Geis, 1969) it is seen that the environment of residues 62 and 108 is affected by the binding of the substrate and the resulting conformational change which the enzyme undergoes (Blake et al., 1967).

The low temperature phosphorescence spectra of the enzyme and enzyme-substrate crystals are shown in Fig. 7. We cannot resolve emission from more than one tryptophan site in either spectrum so that the emission is either from only one site or from more than one site at which the tryptophans are in similar environments. It is clear, however, that the phosphorescence of the complex is sharper with more structure being evident. The ODMR transition frequencies are significantly different for the two crystals and the lysozyme-substrate transitions are much sharper indicating a more homogenous environment for the emitting tryptophan(s). This result is expected when the substrate
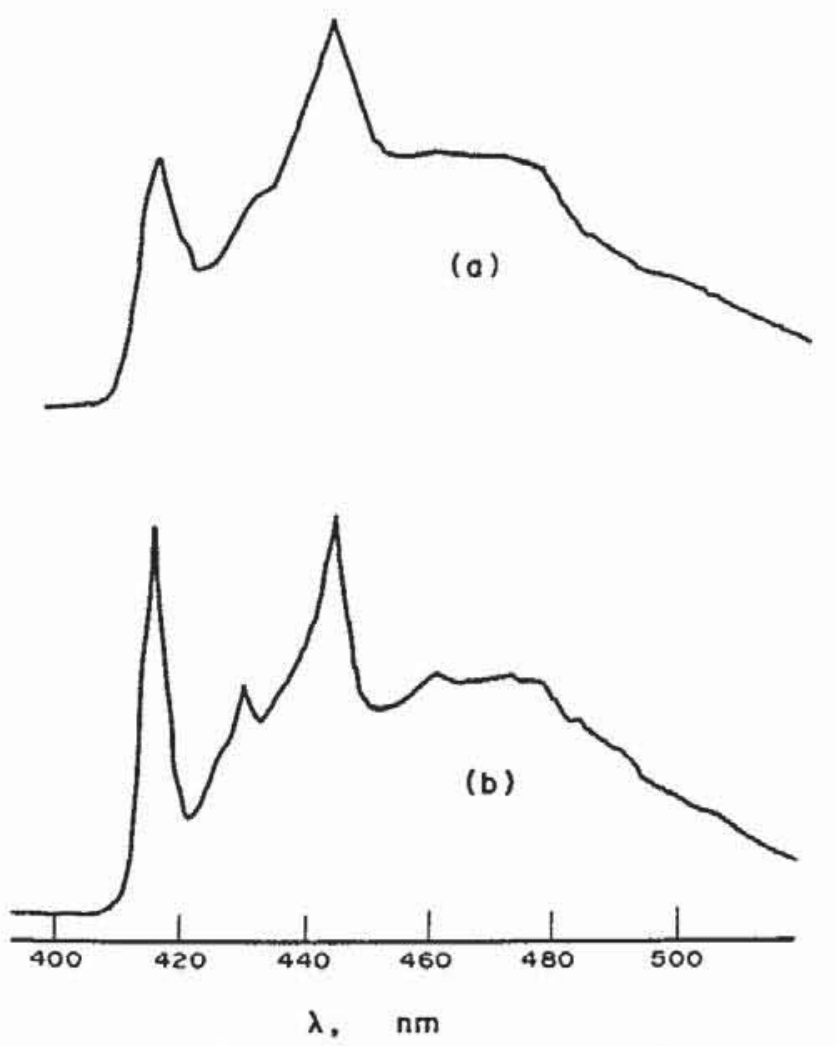

Figure 7. Phosphorescence spectrum of (a), lysozyme and (b), lysozyme complexed with tri-NAG. Both spectra taken at $1 \cdot 3^{\circ} \mathrm{K}$ with an exciting wavelength of $295 \mathrm{~nm}$. cleft is filled and the tryptophan residues are better shielded from the solvent medium.

The ODMR and phosphorescence data for the two crystals are shown in Table 1. The triplet lifetimes, although anomalously shortened in lysozyme relative to most proteins, are not greatly altered upon complexation of the substrate. Thus, as with HLAD the microwave transition frequencies and linewidths (but not the decay lifetimes) are the only parameters whose change with environment we are able to detect with these experiments. It is also noted that in each case, the most red shifted phosphorescence and the highest values of the zero-field parameter $|E|$ are associated with the more highly shielded tryptophan environment. The phosphorescence is more blue shifted and the $|E|$ parameter smaller for free tryptophan in ethylene glycol (EG)- $\mathrm{H}_{2} \mathrm{O}$ than for any of the proteins which have been observed.

\section{CONCLUSIONS}

The primary objectives of identifying different tryptophan sites in a protein and monitoring changes at a given site have been met to a limited extent for the cases discussed above. We have found quantitative correlations between the degree of shielding from the solvent medium (which may be judged by the red shift of the phosphorescence of the emitting site relative to that for free tryptophan in EG- $\mathrm{H}_{2} \mathrm{O}$ ) and the zero-field splitting parameter $|E|$ as well as the linewidths of the ODMR transitions.

In the case of lysozyme as well as several other proteins which we have studied (bovine serum albumin, avidin, apomyoglobin) we could not resolve the emission from different tryptophan sites either optically or by ODMR experiments. However, as discussed by Longworth (1971) the bandwidths of the phosphorescence of most proteins are of the order of $200-300 \mathrm{~cm}^{-1}$, whether the protein has only one tryptophan (human serum albumin, or corticotrophin) or many tryptophans at a variety of sites (lysozyme or avidin). There is, in general, no evidence of heterogenerity of emission from proteins, so that the emission may originate only from one site or from a small number of nearly identical sites.

We have also observed the low temperature $\left(1.3^{\circ} \mathrm{K}\right)$ phosphorescence and ODMR from single crystals of indole (grown from melt by the Bridgeman method after the indole had been zone refined for ca. 200 passes). The phosphorescence bandwidths and ODMR linewidths $(\sim 83 \mathrm{MHz}$ for the $|D|-|E|$ transition) are not appreciably sharper for 
indole crystals than they are for many of the proteins which we have observed. Although it is possible that a broad distribution of environments of emitting indole traps might occur in single crystals leading to the breadth of the phosphorescence bands and ODMR lines, it is more generally found that the emission spectra of crystals are sharp, and originate from discrete traps. Also, the zero-field ODMR lines are generally at least an order of magnitude narrower than found in indole. Thus, it is possible that a larger part of the bandwidths of most protein phosphorescence spectra cannot be attributed to the intrinsic heterogeneity of the environment of the emitting tryptophan chromophores. Experiments are in progress to determine the nature of the line-broadening processes in tryptophan chromophores.

Acknowledgements - This work was partially supported by a U.S. Public Health Service Grant (GM 12327) as well as a NIH Postdoctoral Fellowship (GM 37208 to J. Z.). D. S. received support from the Max Planck Gesellschaft. We wish to thank Professor John A. Rupley for a sample of tri-NAG.

\section{REFERENCES}

Alderton, J. (1946) J. Biol. Chem. 164, 1.

Blake, C., L. Johnson, G. Mair, A. North, D. Phillips and V. Sharma (1967) Proc. Roy. Soc. B167, 378.
Burland, D. M. and J. Schmidt (1971) Molec. Phys. 22, 19.

Dickerson, R., and I. Geis (1969) The Structure and Action of Proteins. Harper \& Row, New York.

Harris, C. B. (1971) J.Chem. Phys. 54, 972.

Harris, C. B. and R. J. Hoover (1971) Chem. Phys. Lett. 12,75 .

Imoto, T., L. S. Forster, J. A. Rupley and F. Tanaka (1972) Proc. Natl.Acad.Sci.U.S.69, 1151.

Kwiram, A. (1967) Chem. Phys. Letts. 1, 272.

Longworth, J. W. (1971) In Excited States of Proteins and Nucleic Acids (Edited by R. F. Steiner and I. Weinryb). Plenum Press, New York.

Purkey, R. M. and W. C. Galley (1970) Biochemistry 9. 3569.

Sharnoff, M. (1967) J.Chem. Phys. 46, 3263.

Schmidt, J., D. A. Antheunis and J. H. van der Waals (1971) Molec. Phys. 22, 1.

Schmidt, J., I. A. M. Hesselmann, M. S. de Groot and J. H. van der Waals (1967) Chem. Phys. Lett. 1, 434.

Schweitzer, D., J. Zuclich and A. H. Maki (1973) Molec. Phys. 25, 193.

Tinti, D. S., M. A. El-Sayed, A. H. Maki and C. B. Harris (1969) Chem. Phys. Lett. 3, 343.

Weinryb, I. and R. F. Steiner (1971) In Excited States of Proteins and Nucleic Acids, (Edited by R. F. Steiner and I. Weinryb). Plenum Press, New York.

Winscom, C. J. and A. H. Maki (1971) Chem. Phys. Lett. 12.264.

Zuclich, J. (1970a) J.Chem. Phys. 52, 3586.

Zuclich, J. (1970b) J.Chem. Phys. 52, 3592.

Zuclich, J., D. Schweitzer and A. H. Maki (1972) Biochem. Biophys. Res. Commun. 46, 1764. 